\title{
A novel charged state prediction method of the lithium ion battery packs based on the composite equivalent modeling and improved splice Kalman filtering algorithm.
}

WANG, S., FERNANDEZ, C., YU, C., FAN, Y., CAO, W. and STROE, D.-I. 
Manuscript Number: POWER-D-20-01101R1

Title: A novel charged state prediction method of the lithium ion battery packs based on the composite equivalent modeling and improved splice Kalman filtering algorithm

Article Type: Research Paper

Keywords: charged state prediction; lithium ion battery pack; composite equivalent modeling; splice Kalman filter; model adaptive; noise correction

Corresponding Author: Dr. Shunli Wang, Ph.D.

Corresponding Author's Institution: Southwest University of Science and Technology

First Author: Shunli Wang, Ph.D.

Order of Authors: Shunli Wang, Ph.D.; Carlos Fernandez, Prof.Dr.; Chunmei Yu, Prof.Dr.; Yongcun Fan, Prof.Dr.; Wen Cao, Prof.; Daniel-Ioan Stroe, Prof.Dr.

Abstract: As the unscented Kalman filtering algorithm is sensitive to the battery model and susceptible to the uncertain noise interference, an improved iterate calculation method is proposed to improve the charged state prediction accuracy of the lithium ion battery packs by introducing a novel splice Kalman filtering algorithm with adaptive robust performance. The battery is modeled by composite equivalent modeling and its parameters are identified effectively by investigating the hybrid power pulse test. The sensitivity analysis is carried out for the model parameters to obtain the influence degree on the prediction effect of different factors, providing a basis of the adaptive battery characterization. Subsequently, its implementation process is carried out including model building and adaptive noise correction that are perceived by the iterate charged state calculation. Its experimental results are analyzed and compared with other algorithms through the physical tests. The polarization resistance is obtained as $\mathrm{Rp}=16.66 \mathrm{~m} \Omega$ and capacitance is identified as $\mathrm{Cp}=13.71 \mathrm{kF}$. The ohm internal resistance is calculated as Ro $=68.71 \mathrm{~m} \Omega$ and the charged state has a prediction error of $1.38 \%$ with good robustness effect, providing a foundational basis of the power prediction for the lithium ion battery packs. 
A novel charged state prediction method of the lithium ion battery packs based on the composite equivalent modeling and improved splice Kalman filtering algorithm

Shunli Wang ${ }^{\text {ab }}$, Carlos Fernandez ${ }^{c}$, Chunmei Yu ${ }^{a}$, Yongcun Fan ${ }^{a}$, Wen Cao ${ }^{a}$, Daniel-Ioan Stroe

${ }^{a}$ School of Information Engineering, Southwest University of Science and Technology, Mianyang 621010, China;

${ }^{b}$ Department of Energy Technology, Aalborg University, Pontoppidanstraede 1119220 Aalborg East, Denmark; ${ }^{c}$ School of Pharmacy and Life Sciences, Robert Gordon University, Aberdeen AB10-7GJ, UK.

Abstract: As the unscented Kalman filtering algorithm is sensitive to the battery model and susceptible to the uncertain noise interference, an improved iterate calculation method is proposed to improve the charged state prediction accuracy of the lithium ion battery packs by introducing a novel splice Kalman filtering algorithm with adaptive robust performance. The battery is modeled by composite equivalent modeling and its parameters are identified effectively by investigating the hybrid power pulse test. The sensitivity analysis is carried out for the model parameters to obtain the influence degree on the prediction effect of different factors, providing a basis of the adaptive battery characterization. Subsequently, its implementation process is carried out including model building and adaptive noise correction that are perceived by the iterate charged state calculation. Its experimental results are analyzed and compared with other algorithms through the physical tests. The polarization resistance is obtained as $R_{p}=16.66 \mathrm{~m} \Omega$ and capacitance is identified as $C_{p}=13.71 \mathrm{kF}$. The ohm internal resistance is calculated as $R_{\mathrm{o}}=68.71 \mathrm{~m} \Omega$ and the charged state has a prediction error of $1.38 \%$ with good robustness effect, providing a foundational basis of the power prediction for the lithium ion battery packs.

Keywords: charged state prediction; lithium ion battery pack; composite equivalent modeling; splice Kalman filter; model adaptive; noise correction

Corresponding author: Shunli Wang. E-mail address: 497420789@qq.com.

\section{Highlights:}

- An improved composite equivalent modeling method is put forward for the lithium ion battery packs.

- A novel splice Kalman filtering algorithm is proposed to realize the charged state prediction.

- The model parameters are identified accurately by investigating the hybrid power pulse test.

- The feature decomposition is conducted by investigating the Bayesian sequential decision strategy. 
The lithium ion batteries are increasing dramatically to the aerospace and automotive application fields, which are used as the powerhouse to provide the uninterrupted energy supply. At the same time, it replaces other batteries gradually and becomes one of the main power sources expanded day by day because of its light weight, high specific energy and no pollution advantages [1]. In order to meet the high power requirements in aerospace and automotive fields, the batteries are usually used in the packing types and taken as the main choices of its power supply application [2-4]. However, it is quite difficult to maintain its energy storage and output safety in the group working application conditions due to the imbalance phenomenon between the internal connected battery cells and the interaction of its series and parallel structure which should be realized online, resulting in its time varying charge-discharge characteristics $[5,6]$. When the large changes occur, the traditional open circuit voltage and Ampere-hour integral methods cannot be used for estimating the charged state value online because of its low accuracy disadvantages, so the improved state estimation methods are urgently needed to improve its prediction effect.

The charged state is one of the core state parameters in the management system of the lithium ion battery packs together with its affiliates in the charged state prediction that should be distinguished from other parameters [7-9]. Since the battery charged state is a physical quantity that cannot be measured directly $[10,11]$, it should be estimated by the information such as voltage, current, internal resistance and temperature that are related to the operating state of the lithium ion battery packs, so its prediction purpose can be turned into a model parameter reorganization problem according to its special impact on the charged state estimation accuracy [12]. Some adaptive estimation methods have been proposed to be suitable for different application backgrounds of the lithium ion batteries [13-16], including electric vehicles, substations, smart grids and financial uninterruptible power systems. These approaches can be divided into three categories: simple calculation, data-driven and battery-based modeling methods [17-20]. The simple calculation ignores the battery internal variation that is involved in different battery types and performs the charged state prediction. It is realized through a simple correspondence model between the external battery parameters, such as the Ampere-hour integration, open circuit voltage and impedance modeling methods [21-25]. The advantage of this typical estimation algorithm is that it does not require the complicated modeling treatment of the battery to be adapted to different charged state levels, making it simple and easy application at any time [26]. Whereas, the disadvantage of this strategy is that its prediction accuracy is not high, which requires the recombination of some other mathematical calculation processes.

The data-driven algorithm is similar to the simple calculation method, the difference of which is that the intelligent modeling method is used to train the relational ones, such as neural network, fuzzy inference, support vector machine and so on [27-30]. The advantage of this 
1 kind of algorithms is that the prediction accuracy is improved and its calculation demand is reduced. Whereas its disadvantage is that a large

3 amount of data is required for its offline model adaptation training that takes quite a long time period. The prediction accuracy is affected

The model-based methods are widely used by the related global experts, especially the Kalman filtering based algorithms. In order to overcome the shortcomings of such charged state estimation algorithms, the extended Kalman filtering and unscented Kalman filtering algorithms are introduced and improved [40]. On the one hand, the ohmic internal resistance is listed as a state variable together with the charged state in order to reduce the modeling error, and the memory-redirected partial least square method is also used to identify the model parameters adaptively that are consistent with the commitments to integrity [41-45]. The impact on the unknown noise is gradually updated but ignored, in which the recursive partial least square method is introduced to identify the battery parameters online to achieve the accurate real-time charged state prediction purpose. And then, the variable variance Kalman filtering method is proposed to update the noise variance in real time in order to reduce the noise interference that results in the inaccurate charged state estimation values [26, 46-48]. The optimal unbiased maximum posterior time-varying estimator can be built to correct the noise statistics online, but the time-varying problem of model parameters cannot be solved effectively due to the accumulated calculation effect. Consequently, the improved Sage-Husa adaptive unscented Kalman filter algorithm is adopted to the prediction system under strict control that is supplied in accordance with its correction strategy [49-51]. As the uncertainty noise of the charged state estimation algorithm is greatly affected by the battery model provided by its step-by-step estimation and correction, the model parameters and state variables can be calculated by the double Kalman filtering processes respectively and the experimental results show that these two algorithms have strong practical characteristics. As a result, the accuracy and robustness can be further improved by the information collected in the real-time working condition power supply process.

The Kalman filtering algorithm can be introduced into the charged state estimation process combined with the open circuit voltage method, which is characterized by the electrical modeling and provides an important phase of a large oscillation with the time-varying temperatures [52]. By fitting the curve relationship between open circuit voltage and charged state, the prediction-measurement-correction strategy can be 
conducted following to the open circuit voltage values as well as its terminal voltage. According to the minimum mean square error principle, the dynamic system can be investigated for the optimal charged state estimation, making changes in the working state monitoring patterns [53]. And then, the obtained state variable can be updated under the actual measured condition to realize the correction and reduce the detection error effect. Its complete phenomenon is known as the closed loop feedback. In order to avoid the introduced noise effectively that is taken place in the state and measurement equations, the Kalman filter algorithm minimizes the charged state estimation errors and conducts the continuous correction for the target accurate value in real time. As a result, the online internal resistance measurement is applied in the capacity and charged state estimation for the lithium ion batteries, in which the prevailing theory predicts that the inner parameters can be characterized by the modeling strategies [54]. The charged state estimation is investigated for the lithium ion batteries based on a novel reduced-order electrochemical model, which could have a major impact on the energy storage systems worldwide and an advanced machine learning algorithm is conducted for the power batteries of diversified drive cycles. As a result, the online charged state estimation algorithm is put forward for the lithium ion batteries by using the improved Kalman filtering theory that is adaptive to the surrounding working conditions.

In order to solve the problem that the unscented Kalman filter algorithm is sensitive to battery model and vulnerable to unknown noise, an improved splice Kalman filtering method is proposed further to improve the charged state prediction accuracy. It also makes the calculation process adaptive to the robust performance of the lithium ion battery packs. Based on the composite equivalent modeling and its correction treatment, the splice Kalman filtering algorithm is realized to estimate the charged state value by using the open circuit voltage characteristic towards different charged state levels combined with the Ampere-hour integration methods, and its prediction accuracy is improved dramatically on the lithium ion battery packs according to the iterate calculation process that represents the mathematical description of the inner electrical variations. Meanwhile, the composite equivalent circuit model is built and its parameters are identified, in which the battery modeling characteristic sensitivity experiment is designed and investigated. The steps of adapting the key parameters are obtained by conducting the dimensional expansion, in which the mean value can be solved by the state equation based on its iterate calculation process. When the latest value of the covariance is used, its linear propagation is conducted directly instead of the unscented transformation, which simplifies the calculation complexity. And then, the noise correction form is built by using the covariance matching and optimal unbiased maximum posterior methods. Finally, the complex working condition tests are investigated along with its experimental consequences, according to which the charged state prediction accuracy and robust performance are improved effectively comparing with the traditional iterate calculation algorithms. 


\subsection{Improved equivalent circuit modeling}

The battery modeling is the mathematical basis of the related working condition monitoring strategy that the battery management system must contend with. At present, the usual lithium ion battery models can be roughly divided into three sorts of categories. The first type is the 10 model obtained by the simplified development of the electrochemical model, which can be also called as the simplified electrochemical model that differs from one model to another. The third kind is the equivalent circuit modeling method that can be trained in ways to realize the accurate charged state estimation, in which an equivalent electrical circuit is used to simulate the external characteristics of the lithium ion battery of competition and influences. After considering the battery polarization effect and modeling complexity fully, the improved equivalent circuit model is constructed, which has simple structure but great influence on the accurate description of the working characteristics for lithium ion battery packs. The related parameters have clear physical meanings, which can simulate the charging and discharging characteristics accurately to meet its stable power supply requirement. The specific circuit is established for the accurate working characteristic expression as shown in Fig. 1.

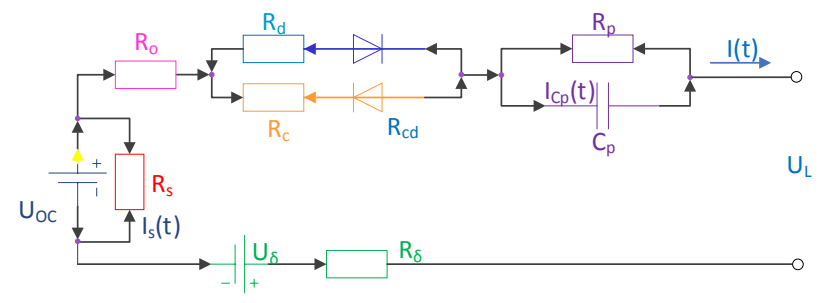

Fig. 1. The improved equivalent circuit model

In Fig. 1, the model assumptions are summarized and listed in detail as follows. $U_{o c}$ represents the open circuit voltage. $R_{\underline{S}}$ is used to illustrate the self-discharge effect, which can be described as the battery degrades effectively to avoid large discrepancies. $R_{o}$ represents the battery ohmic internal resistance. $R_{\underline{\underline{c}}}$ and $R_{\underline{\underline{d}}}$ are the charge and discharge differential resistances, which can be gathered to be $R_{\underline{\underline{c}} \underline{ }}$ for its optimizational calculation. $R_{p}$ and $C_{p}$ are the polarization resistance and capacitance that have great effect on the battery charged state prediction respectively. $I(t)$ is the circuit loop current. $U_{\underline{L}}$ is the load voltage. $U_{p}$ is the polarization voltage that is obtained by the potential

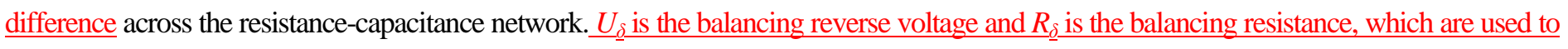
describe the battery cell-to-cell differences. This modeling method can obtain the mathematical expression by different battery effect description, in which the internal resistance is used in the equivalent process with high accuracy characteristics. The series-connected resistance is introduced into the model that is mixed with other circuit components, which is then connected to the parallel reverse diodes to 
The correction treatment is required in the mathematical methods by the iterate calculation process, providing an opportunity for the accurate charged state estimation. Consequently, the electrochemical, neural network and equivalent models are commonly considered in applications, which are strongly tele-connected to the primeval state parameters. The electrochemical model is used to study the influence of internal chemical mechanism and reaction on the model construction process but with high estimation accuracy, which is not suitable for this application due to its large calculation amount. Then, the neural network model is investigated to study how to estimate the energy state value by using the input and output relations to open spaces in the over-lying input factors used for the accurate characterization, which forms many experimental data sequences. Eventually, the estimation error will be large if the data is insufficient which should be escaped due to its dependence on the experimental data. According to Kirchhoff law, the relationship between these parameters can be obtained respectively and accommodated scarcity of the calculation demands as shown in Equation (1).

$$
\left\{\begin{array}{l}
U_{L}=E-I R_{o}-U_{p} \\
I=U_{p} / R_{p}+C_{p} d U_{p} / d t
\end{array}\right.
$$

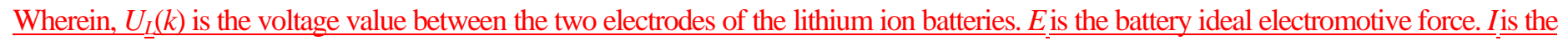
total circulating circuit current of the battery system. $R_{0}$ is the internal resistance. $U_{\underline{p}}$ is the voltage across the polarization resistancecapacitance network, in which $R_{\underline{L}}$ and $C_{\underline{L}}$ are the resistance and capacitance separately. The working characteristics can be revealed by the iterate calculation process of greater transparency by using the basic accumulative calculation treatment, in which the general introduction and calculation requirement are considered in the interact through the mathematical treatment of the equivalent model. According to the accumulation of the environmental influence that erupts into a sudden time point, its charged state is affected by the battery factors such as temperature, charge-discharge state, self-discharge, aging effect etc. The internal chemical reaction is variable and its characteristics are highly nonlinear, which makes the energy state estimation difficult due to the high requirement of the input parameters. There are many charged state estimation methods, in which the ampere-time integration error is large and the open-circuit voltage method is mainly used for the offline energy state estimation. The high-precision methods of the energy state estimation include neural network, fuzzy inference and Kalman filtering that have been studied consciously, in which the Kalman filtering is an optimized autoregressive data processing algorithm. According to the Ampere-hour calculation results and the influence of the surrounding environments with its accurate risk assessment, the charged state definition can be obtained and estimated at external influences as shown in Equation (2).

$$
S(t)=S\left(t_{0}\right)-\eta \int_{t_{0}}^{t} I(t) d t / Q_{N}
$$




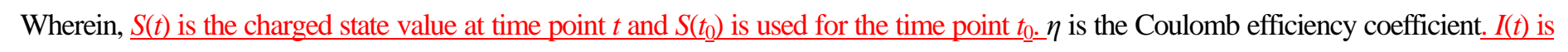

the the current value at time point $t$ and $Q_{N}$ is the battery calibration capacity. Aiming to realize its accurate estimation, the large error disadvantage of the ampere-time integration approach is overcome effectively by combining with the splice Kalman filtering methods.

7 Meanwhile, the open-circuit voltage is mainly used for the correction step, and the high-precision energy state estimation is investigated by combining the thought of neural network, fuzzy reasoning, and Kalman filtering algorithms together, which is an optimized autoregressive 11 data processing method that is quite suitable for the nonlinear working state estimation. Combining the above equations, the discretization process can be used and dissolved to obtain the immune state-space and observation equations. It is used to indicate the working state of the lithium ion battery packs effectively. The calculation process can be described as shown in Equation (3).

$$
\left\{\begin{array}{l}
{\left[\begin{array}{c}
S(k) \\
U_{p}(k)
\end{array}\right]=\left[\begin{array}{cc}
1 & 0 \\
0 & \exp \left(-T_{s} /\left(R_{p} C_{p}\right)\right)
\end{array}\right]\left[\begin{array}{c}
S(k-1) \\
{\left[U_{p}(k-1)\right.}
\end{array}\right]+\left[\begin{array}{c}
-\eta T_{s} / Q_{N} \\
U_{L}\left(1-\exp \left(-T_{s} /\left(R_{p} C_{p}\right)\right)\right)
\end{array}\right] I I(k-1)+\left[\begin{array}{l}
w_{1}(k-1) \\
w_{2}(k-1)
\end{array}\right]} \\
\left.U_{L}(k) E(k)\right]-U_{p}(k)-R_{o} I(k)+v(k)
\end{array}\right.
$$

22

Wherein, $S(k)$ is the charged state value at time point $k$ and $S(k-1)$ is used for the time point $k-1 . U_{p}(k)$ is the voltage value of both point ends to the polarization resistance-capacitance circuit and $U_{p}(k-1)$ is used for the time point $k-1$. In the upper part of Equation (3), two state variables of $S(k)$ and $U_{p}(k)$ are formed into the state equation matrix. $\eta$ is the Coulomb efficiency coefficient. $T_{s}$ is the sampling time interval which is set as $T_{s}=1 \mathrm{~s}$ in the experiment, that is, the sampling frequency is $1 \mathrm{~Hz}$. $Q_{\underline{N}}$ is the calibration battery capacity. $R_{p}$ and $C_{p}$ are the polarization resistance and capacitance. $I(k-1)$ is the current value at time point $k-1 . w_{1}$ and $w_{2}$ are the process noises for both of the amperehour iterate calculation and the polarization effect separately, which are Gaussian white noise with mean value of zero and covariance of $Q$. In the second half of the Equation (3), the observation calculation process is realized. $U_{\underline{L}}(k)$ is the voltage value of the two battery electrodes. The operator $E[S(k)]$ is the battery ideal electromotive force that is described by using the mathematical nonlinear function based on the charged state estimation results, in which the open circuit voltage characteristic curve towards different charged state levels can be obtained corresponding to the charged state variation. $v(k)$ is the observation noise with covariance value of $\boldsymbol{R}$. And then, its aggregated value can be calculated by using the shared precise information along with the charged state estimation process based on the model parameters.

\subsection{Parameter identification considering charge-discharge effect}

The parameters that the model needs to identify include $R_{o}, R_{p}, C_{p}$ and the open circuit voltage characteristic relationship towards different charged state levels, which are all time-varying parameters that change along with the environmental factors such as ambient temperature, charge-discharge rate and battery life degradation in inquiry. In order to obtain the reliable initial value of the model parameters for the charged state prediction algorithm, it is necessary to identify these parameters by means of pulse power discharging experiments, which are implemented to realize the model parameter identification of the lithium ion batteries surrounded by the environmental factors. The 

according to which the anti-phase symmetry effect can be utilized effectively. And then, the model parameters can be calculated by considering both of the charging and discharging processes, making its accuracy to be better. The $1 \mathrm{C}$ constant current discharge is conducted

experimental object is the socialized lithium cobalt oxide batteries for instance. The experimental conditions and processes are designed as follows. Considering the charge-discharge effect, the pulse charge-discharge process of lithium-ion battery packs for the experiment, for 360 seconds and the ambient temperature is set as $25{ }^{\circ} \mathrm{C}$, which is followed by the shelved treatment effectively. The specific pulse charge-discharge process is shown in Fig. 2.

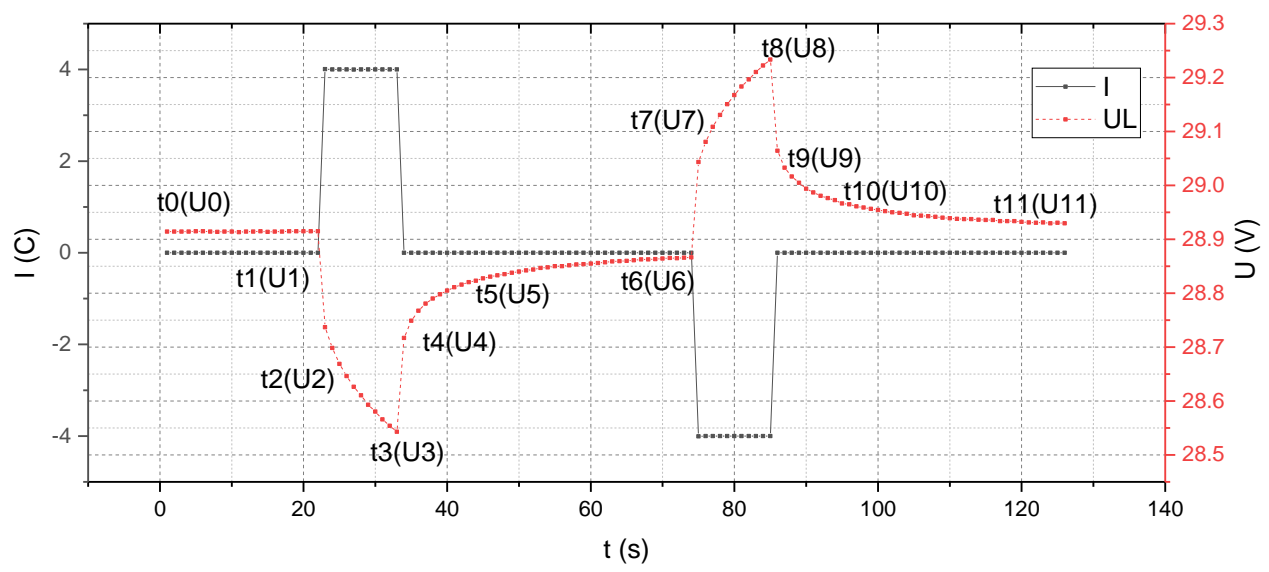

Fig. 2.The specific pulse processes

Wherein, the complete response curve of the terminal voltage is described under the condition of one pulse charge-discharge process, which is recorded as $U_{L}(t)$. $U_{0}-U_{1}$ is the initial stationary stage and $U_{1}-U_{2}$ is the sudden voltage drop stage during the pulse discharging maintenance process. $U_{2}-U_{3}$ is the constant current discharge stage and $U_{3}-U_{4}$ is the terminal voltage changes in the end of the pulse discharging process when the battery pack is not discharged any more during this time point. $U_{4}-U_{5}$ is the zero input response state of the terminal voltage after the battery discharge period ends. The battery terminal voltage basically does not change from the $U_{5}-U_{6}$ phase, which returns to the rest state completely. $U_{4}-U_{3}$ and $U_{8}-U_{9}$ are defined as the potential change of the ohmic internal resistance in the case of power failure, according to which $R_{o}$ can be identified accurately. $U_{6}-U_{4}$ and $U_{9}-U_{11}$ are the voltage change between the two ends of the $\mathrm{RC}$ loop during the discharging and charging processes, and then $R_{p}$ can be identified accurately. The calculation process of these two parameters is shown in Equation (4).

$$
\left\{\begin{array}{l}
R_{o}=\left[\left(U_{4}-U_{3}\right) / I+\left(U_{8}-U_{9}\right) / I\right] / 2 \\
R_{p}=\left[\left(U_{6}-U_{4}\right) /+\left(U_{9}-U_{11}\right) / I\right] / 2
\end{array}\right.
$$

Wherein, $R_{\underline{O}}$ is calculated by the voltage change of considering both of the charging and discharging effects as well as $R_{p}$, so that the parameter identifying accuracy can be improved effectively. The energy state essence of the lithium ion battery pack is estimated by using the 
ampere-time integration method to calculate its value accurately, in which the charge-discharge current is assumed to be measured with high precision. And then, the measured voltage value is used to correct the energy state value that is obtained from the model prediction together with the ampere-hour integration. Consequently, a suitable equivalent battery model is established for the accumulated energy description of the lithium ion batteries, in which the estimation accuracy mainly depends on its mathematical description effects. The battery is regarded as a magnitude power system and the energy state is a state parameter of the system. Meanwhile, the battery charge-discharge current is taken as the input factor of the system and the terminal voltage is used as its output parameter. The system state is updated continuously by the devastating error of the observed terminal voltage and the energy state can be estimated at the nearby time points, thereby the energy state can be generated with minimum-variance characteristics by the iterate calculation treatment. The time parameter should be calculated by using the experimental voltage values of different time points combined with the battery output factor measurement influenced by the surrounding working conditions. The calculation process of the time constant $\tau$ is described as shown in Equation (5).

$$
\tau=\frac{\left(t_{6}-t_{4}\right)}{\ln \left(1-\frac{U_{6}-U_{4}}{U_{1}-U_{4}}\right)}+\frac{\left(t_{11}-t_{9}\right)}{\ln \left(1-\frac{U_{9}-U_{11}}{U_{9}-U_{6}}\right)}
$$

Wherein, $\tau$ is the time constant. $t_{\underline{4}}, t_{\underline{6}}, \underline{t_{11}}$ and $t_{9}$ are the time points corresponding to $U_{4}, U_{6}, \underline{U}_{11}$ and $U_{9}$ as shown in Fig. 2 . $U_{1}$ is the initial voltage before the pulse charge-discharge test. According to the relationship between polarization capacitance $C_{p 2}$ polarization resistance $\underline{R}_{p}$ and time constant $\tau$, the parameter $C_{p}$ can be obtained incapable of producing the great sudden voltage change. By conducting the inspection of the mathematical calculation process, its strict regulations can be complied as well as the qualifications and its mathematical calculation method is shown in Equation (6).

$$
C_{p}=\tau / R_{p}
$$

Wherein, $C_{p}$ is the polarization capacitance. $\tau$ is the time constant and $R_{p}$ is the polarization resistance. The open circuit voltage value can be obtained by fully sinking the terminal voltage that comes up with the electromotive force establishment of the voltage points including $U_{6}$ and $U_{11}$, according to which it is recorded conveniently and recognized as efficient to be $E$ as shown in Equation (7).

$$
E=\left(U_{6}+U_{11}\right) / 2
$$

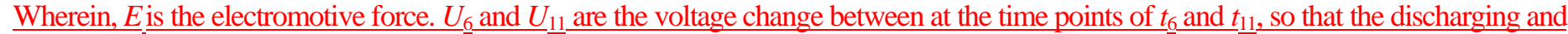
charging influence can be offset by conducting these two symmetric time points. It is a question that the time parameter should be applied to realize the iterate calculation process and establishing the reasonable state and observation equations should be the most critical step of the iterate calculation process, so an accurate battery model must be established to gain its predictive value. However, since the battery system exhibits strong nonlinearity, it is not ideal for this nonlinear energy state estimation system if only taking the basic Kalman filtering theory 
into account. Therefore, the splice Kalman filtering algorithm is put forward to linearizing the nonlinear system through Taylor expansion to forecast its future state. After linearization, the traditional Kalman filtering treatment can be conducted and behaved to estimate the state parameters, according to which the expected variables are estimated, providing a good choice to use double unscented transformation to estimate the energy state value accurately. In addition, when the parameter measuring accuracy is not high and the calculation error is not considered seriously therefore, it is hard to distinguish the energy state that is stored by the battery system. Because this algorithm ignores the higher order terms other than the second-order after the Taylor expansion treatment, its estimation error mainly depends on the correcting calculation process. By analyzing the transient response of the resistance-capacitance structure of the $t_{4}-t_{5}$ phase, $\tau=R_{p} C_{p}$ can be identified and prepared for the steadily built up characteristics, the identification process of which is shown in Equation (8).

$$
U_{L}(t)=E-R_{p} I e^{-t / \tau}
$$

Wherein, $U_{\underline{L}}(k)$ is the voltage value between the two battery electrodes when the lithium ion battery is set to be charging or discharging conditions. $E$ is the battery ideal electromotive force that is calculated by using Equation (7). I is the total circulating circuit current of the battery system. $t$ is the time point and $\tau$ is the time constant. Combined with the recognition of the above equations, the corresponding parameters of the experimental application case can be obtained by the curve fitting treatment, and the varying pattern of the open circuit voltage towards the charged state characteristic is obtained by the experimental cycling hybrid pulse power characterization tests. As for the hybrid pulse power characterization test, the detailed process is designed as follows. Firstly, $1 \mathrm{C}$ constant current discharge is investigated in the environmental temperature conditions of $25^{\circ} \mathrm{C}$, and the discharging time of 360 seconds ensures that the charged state interval between each pulse charge-discharge treatment is 0.1 . And then, the stewing time of 600 seconds ensures that the terminal voltage change rate is less than $1 \mathrm{mV} / \mathrm{min}$. The experimental results of the cyclic pulse discharging maintenance are obtained by the accumulated calculation in small amounts of its discharging energy that are shown in the left part of Fig. 3.

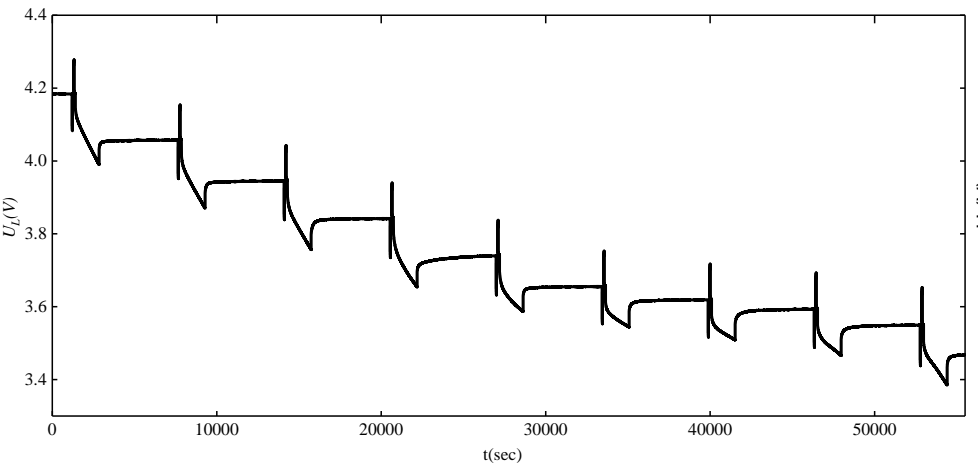

(a) Cyclic pulse discharging maintenance

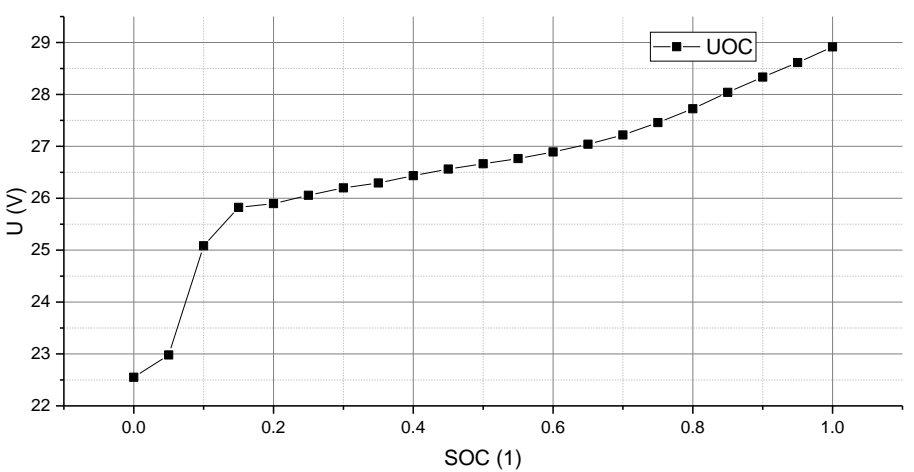

(b) Open circuit voltage characteristics

Fig. 3.The specific pulse discharging process and its open circuit voltage characteristics 
As can be known from the left part of Fig. 3, the open circuit voltage value can be accumulated along with its energy state variation. It is obtained at the time point before each pulse discharge maintenance. Then, the corresponding battery terminal voltage value can be extracted by the discrete data points, according to which the open circuit voltage characteristics towards different charged state levels as shown in the right part of Fig. 3. Thus, the open circuit voltage characteristic towards different charged state levels can be obtained by curve fitting tool of the Matlab software, the functional relationship of which is shown in Equation (9).

$$
E=a_{0}+a_{1} s^{1}+a_{2} s^{2}+a_{3} s^{3}+a_{4} s^{4}+a_{5} s^{5}+a_{6} s^{6}
$$

Wherein, $s$ expresses the charged state and $a_{k}(k=1 \sim 6)$ are the constant and magnitude parameters of this equation by decoding the inner information of the experimental results.

\subsection{Iterative calculation with time-varying internal resistance}

As the unscented Kalman filter algorithm is sensitive to the battery model and susceptible to the interference from unknown noise, the splice Kalman filtering algorithm is put forward to handling these problems well. Its implementation process is introduced in detail, including two parts named as the model adaptation and the noise adaptation. Therefore, the system noise is generally approximated as white noise in accordance with the Gaussian distribution as well as its process noise, the expectation value of which is linearized along with its covariance value of the estimation process. The linear state equation is used to estimate the system state by using the input-output data. Because it is estimated completely at the time domain, there is no mutual conversion to realize the translation treatment for the time domain and the frequency domain, so the calculation amount is quite small but has good real-time estimation effect. The model parameter adaptation is realized by conducting the dimensional prediction in that the key model parameter $R_{o}$ is listed as the state variable that can be estimated together as well as $S$ and $U_{p}$. And then, the state-space equation is modified accordingly as shown in Equation (10).

$$
\begin{aligned}
& \left\{\left[\begin{array}{l}
S(k) \\
U_{p}(k) \\
R_{o}(k)
\end{array}\right]=\left[\begin{array}{ccc}
1 & 0 & 0 \\
0 & \exp \left(-T_{s} /\left(R_{p} C_{p}\right)\right) & 0 \\
0 & 0 & 1
\end{array}\right]\left[\begin{array}{c}
S(k-1) \\
\left.U_{p}(k(k-1))\right) \\
R_{o}(k(k-1))
\end{array}\right]+\left[\begin{array}{c}
-\eta T_{s} / Q_{N} \\
\left.R_{p}\left(1-\exp \left(-T_{s} /\left(R_{p} C_{p}\right)\right)\right)\right) \\
0
\end{array}\right] I(k-1)+\left[\begin{array}{l}
w_{1}(k-1) \\
w_{2}(k-1) \\
w_{3}(k-1)
\end{array}\right]\right. \\
& U_{L}(k)=E[S(k)]-U_{p}(k)-R_{o}(k) I(k)+v(k)
\end{aligned}
$$

Wherein, $S(k)$ and $S(k-1)$ are the charged states at the time points of $k$ and $k-1 . U_{\underline{L}}(k)$ and $U_{\underline{L}}(k-1)$ are the voltage values of the polarization resistance-capacitance circuit at the time points of $k$ and $k-1$. In the upper part of Equation (10), three state variables of $S(k), U_{p}(k)$ and $R_{\underline{o}} \underline{(k)}$ are formed into the state equation matrix. $\eta$ is the Coulomb efficiency coefficient. $\underline{T}_{\underline{S}}$ is the sampling time interval and $Q_{\underline{N}}$ is the calibration capacity. $R_{\underline{L}}$ and $C_{\underline{\underline{ }}}$ are the polarization resistance and capacitance. $I(k-1)$ is the current value at time point $k-1 . R_{\underline{o}}(k)$ and $R_{\underline{o}}(k-1)$ are the internal resistances at the time points of $k$ and $k-1 . w_{1}, w_{2}$ and $w_{3}$ are the calculation process noises for the ampere-hour, polarization effect and internal resistance. In the second half of the Equation (3), the observation calculation process is realized. $U_{L}(k)$ is the voltage value of the 
$\$$ ideal electromotive and $v(k)$ is the observation noise. To estimate the energy state value of the lithium ion battery pack, it is required to be of 5 white noise characteristics for the process and observation noises together with the Gaussian distribution. In this case, the process noise can be facilitated and the covariance of the observed noise is controlled for the estimation process, in which the Taylor expansion is applied to unfold the system model of the lithium ion battery pack. And then, the first-order linearization model is left by removing the high-order terms. After obtaining the linearization model, the energy state of the lithium ion battery pack is further estimated by the traditional Kalman filter, and its calculation algorithm flow can be obtained accordingly. Then, the lithium ion battery packs can release more energy than usual by considering much about its legitimate application. This is because they have been stored since the last common charging treatment, 17

Wherein, $X(k)$ is the state variable matrix at $k$ time point and $U(k)$ is the input matrix by supposing $Y(k)$ as the observation matrix. $f$ and $h$ are the transfer functions, in which $\underline{W}(k)$ and $\underline{V}(k)$ are the system noise and observation noise respectively that can be ascribed to the fact that the default value of them is the uncorrelated Gaussian white noise. The symmetric sampling strategy is selected for the unscented transformation process mainly because of its advantages in adaptive calculation without tedious calculation. The mean and covariance values of the scientific calculation process can match the real value of the third-order Taylor expansion, and it is significantly higher than the accuracy of the extended Kalman filtering algorithm, which is made up largely due to its high correction features.

As for the lithium ion battery packs, the tectonic energy state parameter is affected by the factors such as temperature, charge-discharge current rate, self-discharge and aging effect. The internal chemical reaction is variable and it beneaths the out-detective parameters. Its characteristics are highly nonlinear due to the colliding influence of the lithium ions. Because of these characteristics, the accurate energy state estimation is very difficult and the promotion process of the high-power lithium ion battery packs tends to be pent-up. By the real-time optimal correction of its state variables, the estimation accuracy of the energy state can be improved by combining with the measurement input factors, which performs the first-order linearization on Taylor expansion of the nonlinear functions. The remaining high-order terms are ignored and written off, according to which the nonlinear characteristics are expressed in the precision calculation step [50], so that it can be applied to the nonlinear energy management system. The specific steps are shown as follows. 
(1) As the estimation results are always influenced by the initial value of the parameters, the mean and variance values should be initialized accordingly and this is also the reason why the precious factor initialization should be conducted as the origin charged state through the foundation of the model factors. The related variable initialization can be realized by Equation (12).

$$
\left\{\begin{array}{l}
X_{0}=E[X(0)] \\
P_{0}=E\left[\left(X(0)-X_{0}\right)\left(X(0)-X_{0}\right)^{T}\right]
\end{array}\right.
$$

Wherein, $X_{0}(k)$ is the initial state variable matrix that is obtained by calculating the expected value of $X(0)$. $P_{0}(k)$ is the covariance matrix that is obtained by combining the calculation of $X_{0}$ and $X(0)$ together.

(2) The $2 L+1$ sigma points can be generated and recognized that comes from the experimental data onto the mathematical techniques as a result of the decoding evidences. What is happening to the short-term timescale is that it is actually imposed on a long-term cycling chargedischarge working conditions. And then, the corresponding factor weights can be calculated resulting from the parameter values of the last time point, which are calculated by using the Equation (13).

$$
W_{(i)}^{m}=W_{(i)}^{c}=\left\{\begin{array}{l}
\lambda /(\lambda+L), i=0 \\
\lambda /[2(\lambda+L)], i \neq 0
\end{array}\right.
$$

Wherein, the weight coefficient matrix $W^{m}$ is used to calculate the mean value of the state parameters and $W^{c}$ is used to calculate the covariance matrix. $\lambda$ is the proportional coefficient, which is chosen and used to adjust the distance between the sigma point and $X_{0}$, affecting the deviation caused by the high-order matrices. $L$ represents the dimension of the state variable and its value is 3 . Its relationship satisfies $\lambda+L=3$, the calculation process of which is shown in Equation (14).

$$
\left\{\begin{array}{l}
\hat{X}_{k-1, i}^{+}=\hat{X}_{k-1}^{+}, i=0 \\
\hat{X}_{k-1, i}^{+}=\hat{X}_{k-1}^{+}+\sqrt{(L+\lambda) P_{k-1}^{+}}, 1 \leq i \leq L \\
\hat{X}_{k-1, i}^{+}=\hat{X}_{k-1}^{+}-\sqrt{(L+\lambda) P_{k-1}^{+}},(L+1) \leq i \leq 2 L
\end{array}\right.
$$

Wherein, the prediction value of the state parameter matrix $X$ can be obtained under different conditions by considering the relationship between $i$ and $L$, in which the covariance matrix $P$ is used for the correction treatment of the prediction process. The sequence and size of the data matrices also have great influence on the calculation complexity. In response to the regional demands of the power supply process of the lithium ion battery packs, it can also be fine-tuned in the middle according to the actual situation.

(3) The one-step time updated state variable and its mathematical treatment can be investigated by using the following calculation process. The eventual formality of receiving the calculation result can be obtained in the last calculation step, which has maintained to be strong and 


$$
\left\{\begin{array}{l}
\hat{X}_{k, i}^{-}=f\left(\hat{X}_{k-1, i}^{+}, U(k-1)\right), 0 \leq i \leq 2 L ; \hat{X}_{k}^{-}=\sum_{i=0}^{2 L} W_{(i)}^{m} \hat{X}_{k, i}^{-} \\
P_{k}^{-}=\sum_{i=0}^{2 L} W_{(i)}^{c}\left[\left(\hat{X}_{k, i}^{-}-\hat{X}_{k}^{-}\right)\left(\hat{X}_{k, i}^{-}-\hat{X}_{k}^{-}\right)^{T}\right]+Q
\end{array}\right.
$$

Wherein, the state parameter matrix $X$ is obtained for all the sampling particles, which is then used for the overall state matrix calculation by the weighted calculation using the weight coefficient matrix $W^{m}$. The covariance matrix $P$ is also calculated by the weighted calculation of the weight coefficient matrix $W^{c}$. The purpose of the above equation is to realize the one-step state prediction together with its error covariance matrix calculation. Since the state-space equation is linear, it can be solved by the covariance linear propagation directly away from the traditional calculation treatment. The battery model is suitable for the energy storage and discharge, which makes it difficult to be forecasted accurately. Plus, it is hard to identify the energy stored by the battery at least. As a result, it should be figured out how to effectively put a voltmeter in a battery cell to investigate the charged energy for the first time. The proposed iterate calculation algorithm does not require the nonlinear transmission links to the same model building process, which simplifies the calculation process as shown in Equation (16).

$$
\left\{\begin{array}{l}
\hat{X}_{k, i}^{-}=f\left(\hat{X}_{k-1, i}^{+}, U(k-1)\right) \\
P_{k}^{-}=F P_{k-1}^{+} F^{T}+Q
\end{array}\right.
$$

Wherein, $X$ is the state parameter matrix and $f(*)$ is its nonlinear transformation function by taking the state value and measured parameter value $U(k-1)$ of the last moment as input. $P$ is the covariance matrix. $F$ is the state transition matrix taken into the several calculation process that provides the intellectual underpinning for this important region, according to which the error correction can be realized in the real-time fundamental energy management with effective architecture. $Q$ is the covariance matrix of $W$ for the process noise description.

(4) When it is used to estimate the energy state value of the lithium ion battery packs, the process noise is initialized along with the observation noise to overcome the limitation of the basic Kalman filtering methods. In this case, the process noise can be facilitated to get everything right, and the covariance of the observed noise is controlled. The state variable sigma point can be corrected without lingered suspicions to avoid the native error accumulation effect, which is realized by the calculation treatment as shown in Equation (17).

$$
\left\{\begin{array}{l}
s \hat{X}_{k-1, i}^{+}=\hat{X}_{k-1}^{+} \\
s \hat{X}_{k-1, i}^{+}=\hat{X}_{k-1}^{+}+\sqrt{(L+\lambda) P_{k-1}^{+}}, 1 \leq i \leq L \\
s \hat{X}_{k-1, i}^{+}=\hat{X}_{k-1}^{+}-\sqrt{(L+\lambda) P_{k-1}^{+}},(L+1) \leq i \leq 2 L
\end{array}\right.
$$


Wherein, $s$ is the weight coefficient for the unscented transform and $X$ is the state matrix. $P$ is the covariance matrix. The prediction value of the state parameter matrix $X$ can be obtained under different conditions by considering the relationship between $i$ and $L$, in which the covariance matrix $P$ is used for the correction treatment of the prediction process.

(5) There is a certain error between the true value and the estimated value, the reason is that there is a certain error between all the measured parameter values obtained by the mathematical model and the actual measured values. The observed prediction values and the covariance of the sigma points can be obtained to keep up with the iterate calculation of the charged state monitoring process of its practical treatment, in which the correction maintenance can be made as a confluence that is shown in Equation (18).

Wherein, $Y_{\underline{k} . \underline{i}}$ is used as the observation matrix. $f(*)$ is the nonlinear observation equation transformation function by taking the state value $\underline{X}_{k, i}$ and measurement parameter value $U(k)$ as input. $s$ is the weight coefficient and $X$ is the state matrix. The overall observation matrix value is then calculated by the weighted sum using the weight coefficient matrix $W^{\mathcal{C}} . P_{\underline{y}}$ is the autocorrelation coefficient matrix only for the observation matrix $Y . P_{\underline{x}}$ is the cross correlation coefficient matrix for both of the state matrix $X$ the observation matrix $Y$. $R$ is the covariance matrix of $\boldsymbol{V}$ for the observation noise expression.

(6) Along with more detailed records of the charged state in its energy supply application, the accurate estimation is investigated by conducting the real-time correction treatment to prevent the huge estimation errors. As a result, the Kalman gain is calculated by using the covariance as shown in Equation (19).

$$
K_{k}=P_{x y}\left(P_{y}\right)^{-1}
$$

Wherein, $K_{\underline{k}}$ is the Kalman gain, which is calculated by taking the autocorrelation coefficient matrix $P_{\underline{y}}$ and the cross correlation coefficient $\underline{\text { matrix } P_{x y}}$. It is then used in the correction treatment as shown in Step 7.

(7) The long records are revealing very useful things to express the long-term cycling characteristics, which are described in the mathematical ways and used in the real-time charged state estimation. The state variable measurement and covariance updating treatment can be conducted as shown in Equation (20). 
Wherein, $X_{k}^{+}$is used as corrected state matrix value and $X_{k}^{-}$is used as the state matrix value before correction. In the correction calculation process, the correction amount is obtained by calculating the residual between the calculated value and the predicted value of the observation

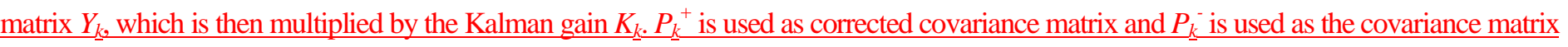
value before correction. During the correction process of the covariance matrix, the correction amount is calculated by using the Kalman gain $\underline{K}_{k}$ and the autocorrelation coefficient matrix $P_{\underline{y}}$

As can be known from Equation (12) to Equation (20), the whole structure of this iterative calculation is a linear combination of the measurement parameters for each step to simplify the estimation process, and the detailed parameters are obtained by the nonlinear correction treatment to correct the accumulated errors as the battery degrades over time by using the time-varying parameters including $R_{0}$ :

\subsection{Noise covariance correction strategies}

In addition to the battery model parameters changing along with the stress conditions, the noise in the actual environment is also uncertain, so it is necessary to adapt the time-varying noise based on the model adaptation, according to which the prediction accuracy of this algorithm is further improved. The algorithm corrects the noise covariance to make it as close as possible to the true noise level and underpins for the next calculation step, and the estimator starts the recursive calculation from the time point of $k=m+1$. When $k<m+1$, the noise can be calculated by the optimal unbiased time-varying estimator statistically, in which $m$ is the window width. According to experience, the window width is set to be $m=20$ and it is particularly suitable for the manifested parameters in the correction process. Then, the noise data is processed by the unbiased time-varying estimator by doing high-quality studies together with its large scale demands, according to which the

Wherein, $z(k)$ is the residual between the measured parameter value of $y(k)$ and its observation value using the previous time point state factor value. $d(k)$ is the correction coefficient weight. $b$ is a forgetting factor that is used to weaken the weight of noise data onto the filtering window, which is conducted with a long-term time period of several hours or more. However, it generally takes a value of 0.98 over the long 
term because it is mostly constant, leaving things to be adaptive. Therefore, combining the system-state at the previous time moment with the error covariance and the Kalman gain, a priori prediction value can be obtained for the expected time period. The two parameter values are true values before and after the one-step calculation, which are not available in the actual working conditions. The posterior state estimation is used at the previous time moment, so it is also necessary to predict the state value of the next step in advance. According to the measurement function of the mathematical model, the measured value is a function of estimating the calculation value, but the estimated value cannot be obtained in actual cases. In the case of the linearization error, its calculated value can be obtained from the mathematical model, which should be equal to the physical value that is directly measured by the management system. It is also named as the measured true value, according to which the linearization error of the mathematical model should be pulsed in the prior estimation step. After that, the parameter $Q$ is estimated by holding accuracy in the iterate calculation process of particular area to check the experimental results, which is described as shown in Equation (22).

$$
Q_{k}=\left(1-d_{k}\right) Q_{k-1}+d_{k}\left(K z_{k} z_{k}^{T} K^{T}+P_{k}^{+}-P_{k}^{-}\right)
$$

Wherein, $Q$ is replaced in the original expression by using the latest prediction value of $Q_{k}$, in which the most common problem of the calculation processes is the balancing treatment for the estimation accuracy and the calculation complexity. There is a certain error between the true value and the estimated value because of the measurement error between all the measured values obtained by the mathematical model compared with the actual measured values. The splice Kalman filtering algorithm is based on the ordinary Kalman filtering algorithm, which is a technique for online linearization that is used to linearize the estimated parameters for performing the iterate calculation treatment, thereby achieving the accurate energy state estimation of the lithium ion battery packs. Therefore, the parameter $R$ is estimated and experienced in the iterate calculation process to avoid its error rising phenomenon above its safety levels, which is described with high complexity as shown in Equation (23).

$$
R_{k}=\left(1-d_{k}\right) R_{k-1}+d_{k}\left(z_{k} z_{k}^{T}-P_{y}\right)
$$

Wherein, $R$ is replaced in the original expression of the latest prediction value of $R_{k}$. When $k \geq m+1$, the noise is estimated in real time by using the proposed special impact correction method. The approximation of the charged state and the terminal voltage covariance is realized by the limited calculation process, in which the estimated value is filled with noise when the measurement error of the closed circuit voltage is large. The correction strategies are developed for serious conditions as the primary components, in which the calculation process is obtained as shown in Equation (24). 

temperature range dynamic operating environment. A state parameter - correction strategy is proposed to realize its stability guarantee, in which the power supply index of the battery system is guaranteed by improving the stability of the dynamic queue. The research objectives and various research contents are considered comprehensively according to the phased research progress requirements of key steps such as theoretical analysis, model construction and experimental verification. $Q$ is the variation in the correction treatment of the process noise and its expression requires different ways to satisfy various calculation purposes, which is shown in Equation (25).

$$
Q_{k}=K C_{k} K^{K}
$$

Wherein, the parameter $Q$ is replaced in the original expression of the latest prediction value of $Q_{k}$, because these parameters are far apart from each other. As a result, it is not only using different types but different expressions. The technical methods of its modeling and state estimation are analyzed, in which the key points of modeling and estimation techniques are mainly addressed by its basic theoretical research. Furthermore, the test environment is established to ensure the scientificity of research through analysis, verification and comparison. As the measurement noise $R$ prediction grasses the calculation with many different factors, the correction is realized with an improved approach conveniently as shown in Equation (26).

$$
R_{k}=C_{k}+\sum_{i=0}^{2 L} W_{(i)}^{c}\left[\hat{Y}_{k, i}-h\left(\hat{X}_{k}^{+}, u_{k}\right)\right]
$$

Wherein, $R$ is replaced in the original expression by using the latest prediction value of $R_{k}$. As the mathematical treatment of Equation (26) has high correction effect on the measurement noise, the calculation restrains quickly with high precision characteristics by the correction accent on these different expressions modeled on the correction step of the iterative calculation process. It is then verified by the pulse power charge-discharge test and the experimental analysis of complex combined working conditions. Its energy and safety management issues are resolved by the theoretical research, simulation analysis and experimental verification from the perspective of energy decay mechanism analysis and state collaborative estimation. According to the full analysis of temperature, aging and internal resistance influence, the characteristic models are established for both of the battery cells and packs respectively, and it is also used to realize the general mathematical description of its external measurable parameters. And then, a multi-constraint model is established for the charged state prediction, according to which the aging mechanism and performance evolution law are further analyzed to gather the prediction results and the modeling database 
1 is updated. Finally, the equivalent simulation process is improved and the estimation method is modified through the experimental analysis of

$\$$ the working conditions to obtain the whole life cycled state prediction and correction strategies.

\section{Experimental analysis}

7

In order to verify the actual prediction effect of the proposed algorithm, the traditional unscented Kalman filter and extended Kalman filter are used for the comparative analysis, in which the specific indicators are the prediction accuracy and robustness. The model parameters and noise statistical characteristics can be estimated in real time by its combined iterate calculation with the special equivalent circuit modeling 14 approach. In this experiment, the lithium cobalt oxide batteries are used as the experimental objects and the pulse cycle discharge at $25^{\circ} \mathrm{C}$ is conducted as the target working conditions. Then, the corresponding discharge process is obtained and analyzed experimentally.

\subsection{Experiment platform design and realization}

In order to obtain parameters such as battery terminal voltage, current and temperature, a battery charging and discharging test and performance test system platform is established. It is mainly composed of the measurement system, charge-discharge control circuit, signal detection circuit, electronic load, incubator and direct current power supply as well as the target lithium ion battery packs. The designed system and platform is described as shown in Fig. 4.

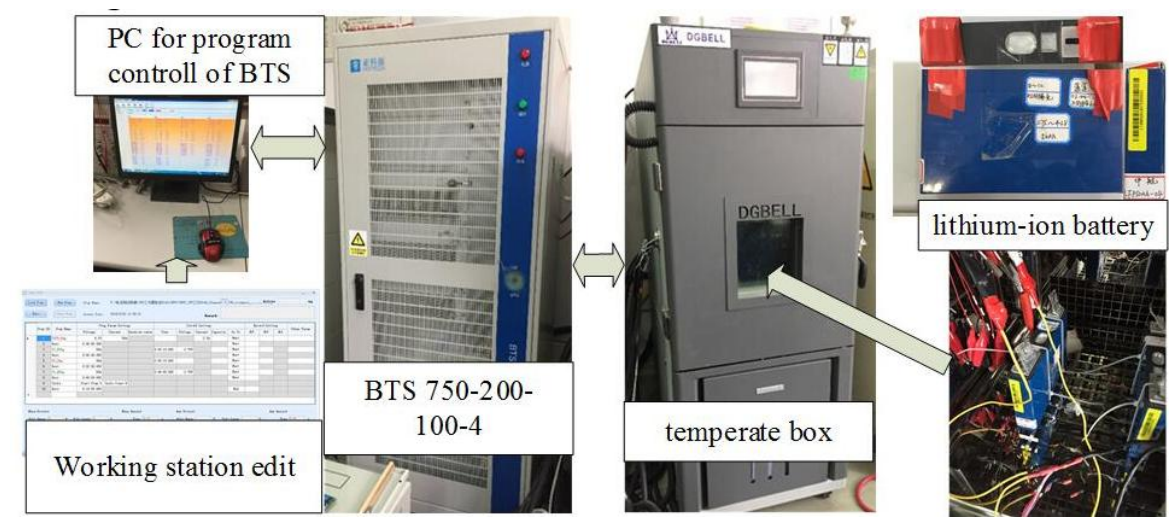

Fig. 4.Experimental platform for the battery pack

The main function of this battery test platform should be realizing the charge-discharge experiments for the lithium ion battery packs of the supposed working conditions, and the collected data is stored at the same time when the experimental test is conducted. The computer sends an instruction in the electronic load through the serial port firstly, which presents its charging and discharging working conditions. Then, the four relay switching states (S1 S4) can be controlled through the digital output function of the data acquisition card with the preset. The working conditions are switched between the charging and discharging states according to the predetermined timing process. Meanwhile, the data onto the current, voltage and ambient temperature can be collected in real time by using the peripheral component inter-connected data 


\subsection{Model parameter identification and correction}

Taking the 7ICP series lithium cobalt oxide battery pack as the experimental object, the hybrid pulse power characterization test is performed to obtain its dynamic characteristics and then the battery model parameters are calculated. In the hybrid pulse power characterization test process, the battery pack is charged with $1 \mathrm{C}$ charging current rate firstly, and then the cyclic hybrid pulse powered characterization tests can be performed on it. The $10 \mathrm{~s}$ pulse discharge process is performed by using the $1 \mathrm{C}$ discharging current. After its

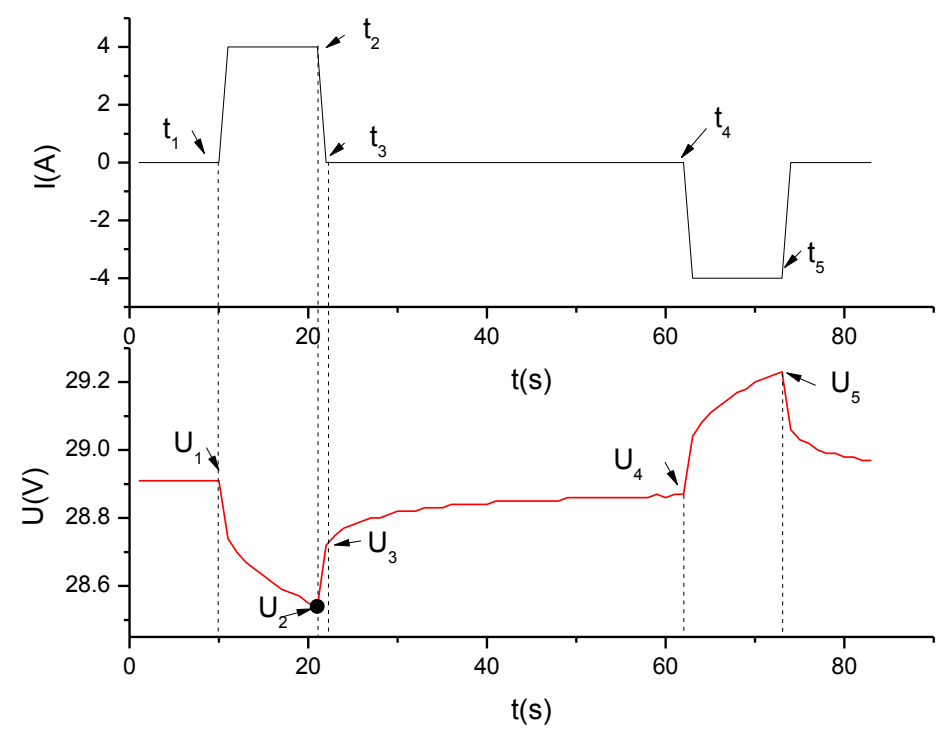

Fig. 5. Hybrid pulse power characterization test

According to the experimental results shown in Fig. 5, the voltage changing curves are analyzed. It is mainly caused by the ohmic internal resistance of the lithium ion battery packs. In addition, the vertical change of the voltage can be described together with its pulse charge- 
1 discharge current. According to the hybrid pulse power characterization test of the expected temperature, the parameter values of the 2

3 equivalent circuit model are calculated as shown in Table 1.

4

5

6

7

Table 1. Model parameters

\begin{tabular}{rllllll}
8 & $\mathbf{S}(\mathbf{1})$ & $\mathbf{U}_{\mathbf{O C}}(\mathbf{V})$ & $\boldsymbol{\tau}(\mathbf{s})$ & $\mathbf{R}_{\mathbf{o}}(\mathbf{m} \mathbf{\Omega})$ & $\mathbf{R}_{\mathbf{P}}(\mathbf{m} \mathbf{\Omega})$ & $\mathbf{C}_{\mathbf{P}}(\mathbf{k F})$ \\
\hline 10 & 1 & 28.91 & 174.05 & 61.8 & 19.09 & 9.12 \\
11 & 0.95 & 28.61 & 179.86 & 61.11 & 18.4 & 10.77 \\
12 & 0.90 & 28.33 & 188.74 & 60.01 & 17.44 & 11.92 \\
13 & 0.85 & 28.04 & 194.78 & 58.23 & 16.34 & 13.52 \\
14 & 0.80 & 27.72 & 206.02 & 58.37 & 15.24 & 13.31 \\
16 & 0.75 & 27.46 & 204.71 & 59.6 & 15.38 & 12.6 \\
17 & 0.70 & 27.22 & 197.22 & 60.15 & 15.66 & 12.86 \\
18 & 0.65 & 27.04 & 199.51 & 60.7 & 15.52 & 11.61 \\
19 & 0.60 & 26.89 & 199.33 & 61.66 & 15.66 & 11.06 \\
21 & 0.55 & 26.76 & 191.3 & 62.35 & 16.48 & 11.3 \\
22 & 0.50 & 26.66 & 191.37 & 65.64 & 17.3 & 12.09 \\
23 & 0.45 & 26.56 & 194.03 & 66.47 & 17.17 & 10.95 \\
24 & 0.40 & 26.44 & 202.62 & 67.84 & 16.75 & 12.2 \\
26 & 0.35 & 26.29 & 208.12 & 69.9 & 16.62 & 12.79 \\
27 & 0.30 & 26.2 & 201.43 & 74.3 & 18.4 & 14.89 \\
28 & 0.25 & 26.06 & 214.38 & 75.94 & 17.58 & 22.2 \\
30 & 0.20 & 25.9 & 221.3 & 77.32 & 17.3 & 26.33 \\
31 & 0.15 & 25.82 & 243.32 & 78.28 & 16.34 & 23.41 \\
32 & 0.10 & 25.08 & 310.94 & 79.1 & 14.01 & 15.79 \\
33 & 0.05 & 22.98 & 415.85 & 88.85 & 17.44 & \\
35 & 0 & 22.55 & 408.33 & 95.31 & & \\
36 & & & & &
\end{tabular}

44 during the charged state prediction process. The polarization internal resistance is established as $R_{p}=16.66 \mathrm{~m} \Omega$ and the polarization 45

As can be known from Table 1, the ohmic internal resistance of the whole hybrid pulse power characterization test process remains almost unchanged when the charged state is greater than 0.3 , and the external polarization internal resistance remains substantially unchanged. The polarization capacitance increases only when the charged state is less than 0.2 . Therefore, the mean value can be taken into the calculation 50 
The functional relationship parameters between the fitted open circuit voltage and charged state are obtained as $A=\left[a_{0}, a_{1}, \ldots, a_{6}\right]=[22.29$, $32.79,-91.07,81.13,61.19,-134.08,56.69]$. The estimation effect verification is performed by the experimental analysis of short-time discharge, which is a combination of different durations and long-term discharge conditions. Furthermore, the comparison of the simulation results from complex working conditions and the estimation result analysis is carried out under different noise effects, and the modeling effect is reliably verified in combination with the balance state correction effect experiments.

\subsection{Charged state prediction results under complex current working conditions}

The experimental object is the battery packs in which 7 lithium cobalt oxide lithium ion battery cells are connected altogether serially with a capacity of $4 \mathrm{Ah}$ as an exportable commodity. Firstly, the battery pack is fully charged with $1 \mathrm{C}$ current rate for the temperature of $25^{\circ} \mathrm{C}$. After standing for one hour, the battery pack reaches a steady state which is set as an integral part of the energy supply system. The simulation experiment is carried out with a parameter sampling interval of $1 \mathrm{~s}$. The parameters used for the simulations are obtained by conducting the realistic experimental tests. Relevant simulation experiments are carried out and analyzed to obtain the estimation effect. The experiments are designed and realized, including instrument inspection, ignition and emergency output of each stage in the application of working conditions. The experimental test process is shown in Fig. 7. 
The charged state value of the battery pack is estimated and compared with the actual value, according to which the prediction result is obtained. The analysis charts show that the proposed splice Kalman filtering algorithm applied to the voltage traction follows the voltage of the terminal voltage variation on the lithium ion battery packs with high precision under current varying conditions, and the coincidence degree with the terminal voltage curve in the actual state is higher than the traditional extended Kalman filtering and unscented Kalman filter methods, indicating that it is suitable to be applied to the charged state prediction of the battery pack based on the natural patterns of high adaptation. The complex current variation experimental tests are conducted under two different conditions named as $\mathrm{C} 1$ and $\mathrm{C} 2$. The Experimental conditions for $\mathrm{C} 1$ is set as $T a=50, T b=5, T c=20, T d=10, T e=30$, which are set as $T a=30, T b=5, T c=20, T d=10, T e=80$ for C2. The prediction results of the complex current variation experimental tests can be obtained with high accuracy as shown in Fig. 8 .
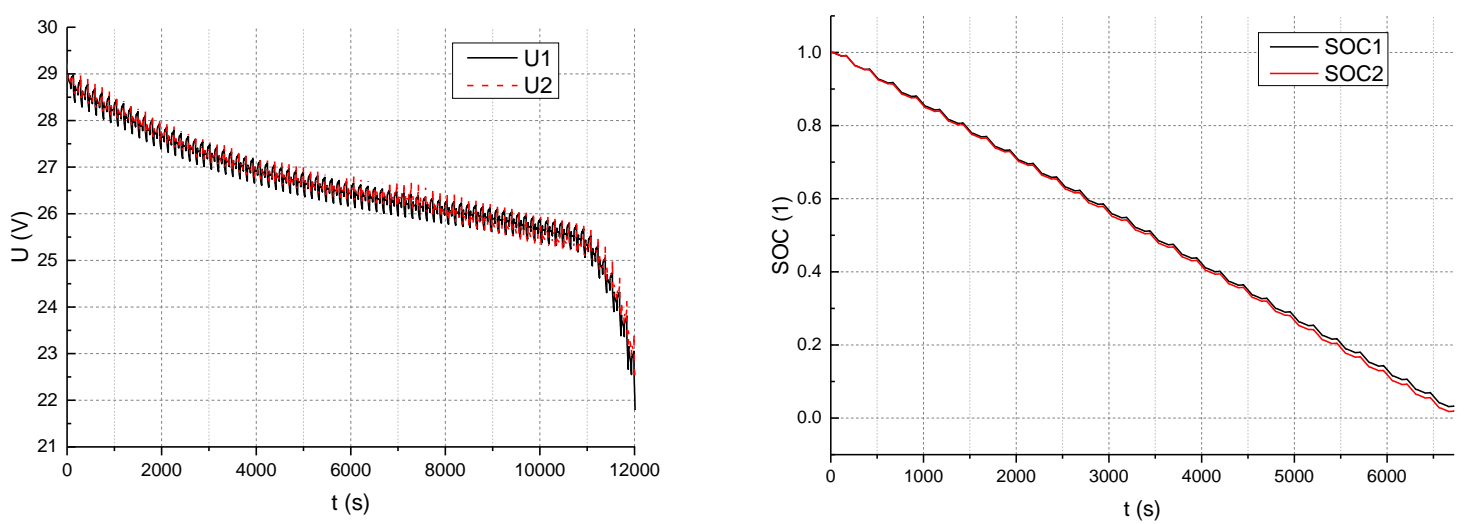


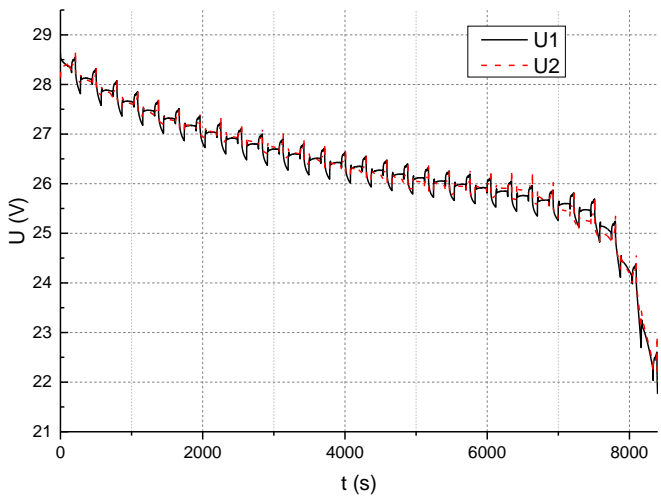

(c) Voltage tracking effect for $\mathrm{C} 2$

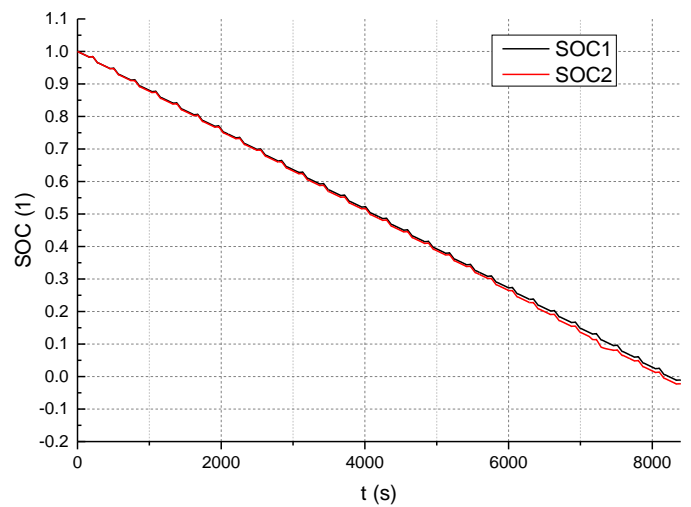

(d) Charged state estimation effect for $\mathrm{C} 2$

Fig. 8.Terminal voltage tracking and charged state prediction curve

As can be seen from Fig. 8, the charged state curve estimated by the proposed algorithm coincides with the actual charged state curve completely. It has the same relationship with the references when the estimation process is conducted by using the Gauss-Hermite particle filter and extended Kalman filter techniques as well as considering its teperature-compensated modeling treatment. By observing the estimation error between the estimated charged state and its actual value, it can be concluded that the error of the charged state prediction is within $1.38 \%$ and its accuracy is greatly improved compared with the traditional extended Kalman filtering and unscented Kalman filter methods. As can be known from the experimental results, current density and voltage have been considered fully and have little influence on the estimation accuracy or robustness when they are employed into the charge-discharge process of the lithium-ion battery pack for the experiment. The temperature influence and effect will be considered in the ongoing research, which will be described by using the temperature correction factors. The temperature change law can be obtained with time-varying environmental conditions in the thermostat equipment shown in Fig. 4. And then, the temperature correction factors can be obtained for the real-time correction.

\section{Conclusion}

The charged state is estimated accurately at the packing lithium ion batteries and its prediction error is reduced greatly by combining the equivalent circuit model and the improved splice Kalman filtering algorithm, which has a good prospect of complex working conditions. Based on equivalent circuit model analysis, the battery modeling and parameter identification are completed, in which the model parameter sensitivity is improved and the influence degree of each parameter on charged state prediction is considered effectively. The influence of the varying battery model parameters on the charged state prediction is different, in which the ohmic internal resistance is the most critical parameter that is followed by the effects of the polarization resistance and capacitance as a potential treatment. Considering the time-varying model parameters and noise interference effects, its prediction accuracy is improved significantly and its robust performance is excellent. It 
1 not only provides a mathematical supply side of the knowledge creation, but also responds to the demand side of societal challenges to the

3 security measures in the packing lithium ion battery energy management.

\begin{tabular}{|c|c|c|c|}
\hline Symbol & Description & Symbol & Description \\
\hline$U_{o c}$ & open circuit voltage & $\underline{E}$ & ideal electromotive force \\
\hline$\underline{R}_{s}$ & self-discharge effect & $\underline{S(t)}$ & charged state value at time point $t$ \\
\hline$R_{o}$ & ohmic internal resistance & $\underline{S\left(t_{0}\right)}$ & charged state value for the time point $t_{0}$ \\
\hline$\underline{R}_{c}$ & charge differential resistance & $\underline{\eta}$ & Coulomb efficiency coefficient \\
\hline$\underline{R}_{d}$ & discharge differential resistance & $Q_{N}$ & battery calibration capacity \\
\hline$\underline{R}_{c d}$ & optimizational calculation of $\underline{R}_{c}$ and $R_{d}$ & $\underline{w(k)}$ & process noise with covariance value of $\boldsymbol{Q}$ \\
\hline$I(t)$ & circuit loop current at time point $t$ & $\underline{v(k)}$ & observation noise with covariance value of $\boldsymbol{R}$ \\
\hline$\underline{U}_{L}$ & load voltage & $\underline{\tau}$ & time constant \\
\hline$\underline{U}_{p}$ & polarization voltage & $\underline{\underline{T}}$ & sampling time interval \\
\hline$\underline{U}_{\delta}$ & balancing reverse voltage & $\underline{R_{\delta}}$ & balancing resistance \\
\hline
\end{tabular}

\section{Acknowledgments}

This research was sponsored by National Natural Science Foundation (No. 61801407) and China Scholarship Council (No. 201908515099). CF would like to express his gratitude to PALS for its support.

\section{References}

O'Malley, R., L. Liu, and C. Depcik, Comparative study of various cathodes for lithium ion batteries using an enhanced Peukert capacity model. Journal of Power Sources, 2018. 396: p. 621-631.

Zhu, J.G., et al., An improved electro-thermal battery model complemented by current dependent parameters for vehicular low temperature application. Applied Energy, 2019. 248: p. 149-161.

$\mathrm{Wu}, \mathrm{B}$. and $\mathrm{W}$. Lu, A consistently coupled multiscale mechanical-electrochemical battery model with particle interaction and its validation. Journal of the Mechanics and Physics of Solids, 2019. 125: p. 89-111.

Jiang, K., et al., Parameter sensitivity analysis and cathode structure optimization of a non-aqueous Li-O-2 battery model. Journal of Power Sources, 2020. 451.

Tang, X.P., et al., A novel framework for Lithium-ion battery modeling considering uncertainties of temperature and aging. Energy Conversion and Management, 2019. 180: p. 162-170.

Li, Y., et al., Development of a degradation-conscious physics-based lithium-ion battery model for use in power system planning studies. Applied Energy, 2019. 248: p. 512-525.

Tang, X.P., et al., Load-responsive model switching estimation for state of charge of lithium-ion batteries. Applied Energy, 2019. 238: p. 423-434.

$\mathrm{Mu}, \mathrm{W} . Y .$, et al., Numerical simulation of the factors affecting the growth of lithium dendrites. Journal of Energy Storage, 2019. 26.

Liu, S.H., et al., Experimental and simulation study on thermal characteristics of 18,650 lithium-iron-phosphate battery with and without spot-welding tabs. Applied Thermal Engineering, 2020. 166.

0. Liu, C., Y.J. Wang, and Z.H. Chen, Degradation model and cycle life prediction for lithium-ion battery used in hybrid energy storage system. Energy, 2019. 166: p. 796-806.

11. Heidari, M., et al., Integrated battery model in cost-effective operation and load management of grid-connected smart nanogrid. Iet Renewable Power Generation, 2019. 13(7): p. 1123-1131.

2. Chen, Z.W., et al., Online state of charge estimation of Li-ion battery based on an improved unscented Kalman filter approach. Applied Mathematical Modelling, 2019. 70: p. 532-544.

13. Degla, A., et al., Comparison study and parameter identification of three battery models for an off-grid photovoltaic system. International Journal of Green Energy, 2019. 16(4): p. 299-308. 
Blondel, P., et al., Nonlinear Circle-Criterion Observer Design for an Electrochemical Battery Model. Ieee Transactions on

$2 \quad$ Control Systems Technology, 2019. 27(2): p. 889-897.

3 15. Bizeray, A.M., et al., Identifiability and Parameter Estimation of the Single Particle Lithium-Ion Battery Model. Ieee Transactions on Control Systems Technology, 2019. 27(5): p. 1862-1877.

6 2019. 167: p. 661-669.

7 17. Liu, K.L., et al., Gaussian Process Regression With Automatic Relevance Determination Kernel for Calendar Aging Prediction of Lithium-Ion Batteries. Ieee Transactions on Industrial Informatics, 2020. 16(6): p. 3767-3777.

8. Wang, R.R. and H.L. Feng, Lithium-ion batteries remaining useful life prediction using Wiener process and unscented particle filter. Journal of Power Electronics, 2020. 20(1): p. 270-278.

19. Zhou, D., et al., Research on online estimation of available capacity of lithium batteries based on daily charging data. Journal of Power Sources, 2020. 451.

Zheng, C.W., Z.Q. Chen, and D.Y. Huang, Fault diagnosis of voltage sensor and current sensor for lithium-ion battery pack using hybrid system modeling and unscented particle filter. Energy, 2020. 191.

Wang, H.Y., et al., Novel estimation solution on lithium-ion battery state of charge with current-free detection algorithm. IET Circuits Devices \& Systems, 2019. 13(2): p. 245-250.

22. Bako, Z.N., et al., Experiment-Based Methodology of Kinetic Battery Modeling for Energy Storage. Ieee Transactions on Industry Applications, 2019. 55(1): p. 593-599.

23. Zhang, Q., et al., Fractional calculus based modeling of open circuit voltage of lithium-ion batteries for electric vehicles. Journal of Energy Storage, 2020. 27.

24. Xuan, D.J., et al., Real-time estimation of state-of-charge in lithium-ion batteries using improved central difference transform method. Journal of Cleaner Production, 2020. 252.

25. Xu, W., J.L. Xu, and X.F. Yan, Lithium-ion battery state of charge and parameters joint estimation using cubature Kalman filter and particle filter. Journal of Power Electronics, 2020. 20(1): p. 292-307.

26. Chen, Z.H., et al., Particle filter-based state-of-charge estimation and remaining-dischargeable-time prediction method for lithium-ion batteries. Journal of Power Sources, 2019. 414: p. 158-166.

27. Wang, W.W., et al., Safety performance and failure prediction model of cylindrical lithium-ion battery. Journal of Power Sources, 2020. 451.

28. Wang, S.L., et al., A novel energy management strategy for the ternary lithium batteries based on the dynamic equivalent circuit modeling and differential Kalman filtering under time-varying conditions. Journal of Power Sources, 2020.450.

29. Wang, L.B., et al., Deformation and failure behaviors of anode in lithium-ion batteries: Model and mechanism. Journal of Power Sources, 2020. 448.

30. Song, Z.Y., et al., The sequential algorithm for combined state of charge and state of health estimation of lithium-ion battery based on active current injection. Energy, 2020. 193: p. 66-77.

31. Song, Y.C., et al., A hybrid statistical data-driven method for on-line joint state estimation of lithium-ion batteries. Applied Energy, 2020. 261.

32. Singh, K.V., H.O. Bansal, and D. Singh, Hardware-in-the-loop Implementation of ANFIS based Adaptive SoC Estimation of Lithium-ion Battery for Hybrid Vehicle Applications. Journal of Energy Storage, 2020. 27.

33. Shang, Y.L., et al., Modeling and analysis of high-frequency alternating-current heating for lithium-ion batteries under lowtemperature operations. Journal of Power Sources, 2020. 450.

34. Qiu, X.H., W.X. Wu, and S.F. Wang, Remaining useful life prediction of lithium-ion battery based on improved cuckoo search particle filter and a novel state of charge estimation method. Journal of Power Sources, 2020. 450.

35. Maheshwari, A., et al., Optimizing the operation of energy storage using a non-linear lithium-ion battery degradation model. Applied Energy, 2020. 261.

Liu, Y., et al., Interface equilibrium modeling of all-solid-state lithium-ion thin film batteries. Journal of Power Sources, 2020. 454.

Feng, Y., et al., Robust Estimation for State-of-Charge and State-of-Health of Lithium-Ion Batteries Using Integral-Type Terminal Sliding-Mode Observers. Ieee Transactions on Industrial Electronics, 2020. 67(5): p. 4013-4023.

Shen, Y.Q., Improved chaos genetic algorithm based state of charge determination for lithium batteries in electric vehicles. Energy, 2018. 152: p. 576-585.

Tang, X.P., et al., A fast estimation algorithm for lithium-ion battery state of health. Journal of Power Sources, 2018. 396: p. 453-458.

Hoang, K.D. and H.H. Lee, Accurate Power Sharing With Balanced Battery State of Charge in Distributed DC Microgrid. IEEE Transactions on Industrial Electronics, 2019. 66(3): p. 1883-1893.

41. Wang, F.K. and T. Mamo, A hybrid model based on support vector regression and differential evolution for remaining useful lifetime prediction of lithium-ion batteries. Journal of Power Sources, 2018. 401: p. 49-54.

Wang, S.L., et al., Open circuit voltage and state of charge relationship functional optimization for the working state monitoring of the aerial lithium-ion battery pack. Journal of Cleaner Production, 2018. 198: p. 1090-1104. 
1 43. Wassiliadis, N., et al., Revisiting the dual extended Kalman filter for battery state-of-charge and state-of-health estimation: A

44. Wei, Z.B., et al., Online monitoring of state of charge and capacity loss for vanadium redox flow battery based on autoregressive exogenous modeling. Journal of Power Sources, 2018. 402: p. 252-262.

45. Xiong, R., et al., A Double-Scale, Particle-Filtering, Energy State Prediction Algorithm for Lithium-Ion Batteries. Ieee Transactions on Industrial Electronics, 2018. 65(2): p. 1526-1538.

7 46. Das, A., T.R. Ashwin, and A. Barai, Modelling and characterisation of ultrasonic joints for Li-ion batteries to evaluate the 8 impact on electrical resistance and temperature raise. Journal of Energy Storage, 2019. 22: p. 239-248.

9 47. Deng, Y.W., et al., Feature parameter extraction and intelligent estimation of the State-of-Health of lithium-ion batteries. 10 Energy, 2019. 176: p. 91-102.

48. Guo, F., et al., A multi-scale parameter adaptive method for state of charge and parameter estimation of lithium-ion batteries using dual Kalman filters. Energy, 2019. 178: p. 79-88.

49. Guo, P.Y., Z. Cheng, and L. Yang, A data-driven remaining capacity estimation approach for lithium-ion batteries based on charging health feature extraction. Journal of Power Sources, 2019. 412: p. 442-450.

50. Homan, B., et al., A realistic model for battery state of charge prediction in energy management simulation tools. Energy, 2019. 171: p. 205-217.

51. Li, X.Y., et al., State-of-health estimation for Li-ion batteries by combing the incremental capacity analysis method with grey relational analysis. Journal of Power Sources, 2019. 410: p. 106-114.

52. Mehrjerdi, H. and R. Hemmati, Modeling and optimal scheduling of battery energy storage systems in electric power distribution networks. Journal of Cleaner Production, 2019. 234: p. 810-821.

53. Li, X.Y., Z.P. Wang, and L. Zhang, Co-estimation of capacity and state-of-charge for lithium-ion batteries in electric vehicles. Energy, 2019. 174: p. 33-44.

54. Xiao, B., Y.G. Liu, and B. Xiao, Accurate State-of-Charge Estimation Approach for Lithium-Ion Batteries by Gated Recurrent Unit With Ensemble Optimizer. Ieee Access, 2019. 7: p. 54192-54202. 


\section{Credit Author Statement}

Shunli Wang: Conceptualization, Methodology, Software

Carlos Fernandez: Writing- Reviewing and Editing.

Chunmei Yu: Data curation, Writing- Original draft preparation.

Yongcun Fan: Visualization, Investigation.

Wen Cao: Supervision.

Daniel-Ioan Stroe: Software, Validation. 


\section{Declaration of interests}

$\bigotimes$ The authors declare that they have no known competing financial interests or personal relationships that could have appeared to influence the work reported in this paper.

$\square$ The authors declare the following financial interests/personal relationships which may be considered as potential competing interests:

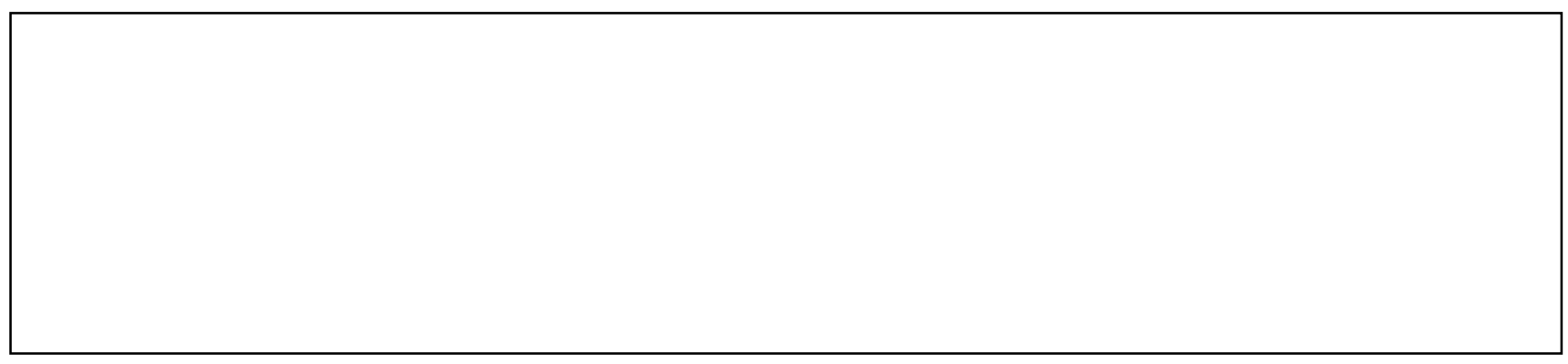

